\title{
Ultra High Speed Semiconductor Electrooptic Modulator Devices for Gigahertz Operation in Optical Communication Systems
}

\author{
Abd El-Naser A. Mohamed, Mohamed A. Metawe'e, Ahmed Nabih Zaki Rashed", Amira I. M. Bendary \\ Electronics and Electrical Communications Engineering Department, Faculty of Electronic Engineering, Menouf, 32951, \\ Menoufia University, Egypt
}

\begin{abstract}
The effects of electrodes geometry and temperature on high frequency radio frequency transmission characteristics are deeply investigated against semiconductor material based electro optic modulator devices such as aluminum gallium arsenide (AlGaAs) and optical waveguide parameters. On the other hand, we have developed the optimization of the electro-optic modulator parameters where the effective index plays an essential role in the evaluation of the bandwidth structure. Therefore, a theoretical analysis of the capacitance, the characteristic impedance and the effective index determine how to increase the bandwidth. The effects of design parameters on the modulating voltage and optical bandwidth are also investigated for different materials based electro-optic modulators by using rigorous transmission modeling techniques. The low-loss wide-bandwidth capability of optoelectronic systems makes them attractive for the transmission and processing of microwave signals, while the development of high capacity optical communication systems has required the use of microwave techniques in optical transmitters and receivers. These two strands have led to the development of the research area of microwave photonics.
\end{abstract}

Keywords Electrooptic Modulator, Semiconductor Material, Optical Bandwidth, High Transmission Performance

\section{Introduction}

Present communication technology relies on fiber-optic systems which include light sources such as a laser, optical fiber, integrated optical components such as modulators and switches, and optical detectors. The lasers and detectors are fabricated using semiconductor materials, and the integrated optical components are generally fabricated using electrooptic single crystal materials such as lithium niobate $\left(\mathrm{LiNbO}_{3}\right)$. Among the integrated optical components, the contribution from electrooptic modulators using $\mathrm{LiNbO}_{3}$ waveguide structures has been significant in the last several decades due to their high speed and chirp-free nature[1]. The essential requirements for efficient electrooptic modulation are low half-wave(switching) voltage and broad 3-dB bandwidth. The optical modulator is a key component for photonics. Optical fiber communications, microwave photonics, instrumentation, and optical signals processing all require optical modulators. Several different technology platforms can be used for the realization of optical modulators. Of these, $\mathrm{LiNbO}_{3}$ based ferroelectric electrooptic modu-

* Corresponding author:

ahmed_733@yahoo.com (Ahmed Nabih Zaki Rashed)

Published online at http://journal.sapub.org/optics

Copyright (C) 2011 Scientific \& Academic Publishing. All Rights Reserved lators provide the most mature technology. Electro-optic polymers and compound semiconductors are also attractive technologies for optical modulators. High-speed integrated electro-optic modulators and switches are the basic building blocks of modern wideband optical communications systems and represent the future trend in ultra-fast signal processing technology. As a result, a great deal of research effort has been devoted to developing low-loss, efficient and broadband modulators in which the radio frequency signal is used to modulate the optical carrier frequency[2]. Most of the work done in the area of designing electrooptic modulators has been strongly focused on using $\mathrm{LiNbO}_{3}[3]$. Interest in research in this field has arisen as lithium niobate devices have a number of advantages over others[4], including large electro-optic coefficients, low drive voltage, low bias drift, zero or adjustable frequency chirp, and the facility for broadband modulation with moderate optical and insertion losses and good linearity. However, on the other hand, $\mathrm{LiNbO}_{3}$ devices cannot be integrated with devices fabricated using other material systems such as semiconductors and as a result they are best suited to external modulation applications. However, with the recent developments in semiconductor technology, modulators based on semiconductor materials have been receiving increasing attention. In particular, Al$\mathrm{GaAs} / \mathrm{GaAs}$ material offers the advantage of technological maturity and potential monolithic integration with other 
optical and electronic devices in creating better optoelectronic integrated circuits(OEIC). Recently, electrooptic polymer modulators have also emerged as alternatives for optical modulators, particularly for low-cost applications for the next-generation metro and optical access systems. Today $2.5 \mathrm{~Gb} / \mathrm{s}$ and $10 \mathrm{~Gb} / \mathrm{s}$ modulators are standard commercial products and $40 \mathrm{~Gb} / \mathrm{s}$ modulators are also being developed for the market after successful prototype demonstrations: however, the continuous demand to increase the data rate further will push their operating frequency well into the millimeter wave range[5].

High-speed optical modulators are essential for dealing with the current explosive growth in network traffic. These modulators are based on two types of physical effect: the electro absorption (EA) effect and the electro-optic (EO) effect. EA modulators have been widely used in recently developed 2.5 and $10 \mathrm{Gbit} / \mathrm{s}$ optical transmission systems, because they have a small driving voltage of around 2 Volt [6], a small size and they are easily integrated with laser sources. However, it is difficult to apply EA modulators to high-bit-rate transmission systems because of their chirp characteristics. EA modulators can only be applied to very-short-reach (VSR) systems at $40 \mathrm{Gbit} / \mathrm{s}[7,8]$. In contrast, the interferometric Mach Zehnder (MZ) modulator provides more flexibility than the EA modulator in terms of chirp controllability. Moreover, MZ modulators are capable of controlling the optical phase. The MZ modulator is the most widely used device employing the EO effect. The optical phase can be controlled by supplying the EO waveguides with a voltage[8]. High-bit rate transmission systems require a phase controlled modulation format, such as a carrier-suppressed return-to-zero(CS-RZ) or optical duo binary signal format[9-11]. These formats are capable of improving the transmission performance because of their dispersion tolerance resulting from the fact that there is less spectral width broadening during modulation.

In the present study, Aluminum gallium arsenide(AlGaAs) semiconductor material based electrooptic external modulators have been developed for extensive use in high speed and long distance optical fiber transmission systems. This is because they can offer the advantages of modulation exceeding multi Gbit/sec combined with a low driving voltage, and they can eliminate the dynamic laser wavelength chirping which limits the span-rate system product due to their fiber dispersion characteristics. Modulators fabricated on semiconductor substrates such as (AlGaAs) and silicon doped materials are particularly attractive in that these exists the possibility of monolithic integration of these devices with other optoelectronic components.

\section{Modeling Analysis}

For $\mathrm{Al}_{\mathrm{x}} \mathrm{Ga}_{(1-\mathrm{x})} \mathrm{As}$, the parameters required to characterize the ambient temperature and operating signal wavelength dependence of the refractive-index, where Sellmeier empirical equation is under the form of[12]:

$$
n^{2}=C_{1}+\frac{C_{2}}{\lambda^{2}-C_{3}}-C_{4} \lambda^{2}
$$

The set of the parameters is recast and dimensionally adjusted as the following $[8,9]: \mathrm{C}_{1}=10.906-2.92 \mathrm{x}, \mathrm{C}_{2}=0.97501$, $\mathrm{C}_{3}=\mathrm{c}_{3} \mathrm{~T}^{2} ; \mathrm{c}_{3}=\left(0.52886-0.735 \mathrm{x} / \mathrm{T}_{\mathrm{o}}\right)^{2}$, for $\mathrm{x}<0.36$. And $\mathrm{C}_{4}=\mathrm{c}_{4}$ $\left(0.93721+2.0857 \times 10^{-4} \mathrm{~T}\right) ; \mathrm{c}_{4}=0.002467(1.14 \mathrm{x}+1)$. We have taken into account the value of $x=0.2$, then the first and second differentiation of above empirical equation with respect to operating optical signal wavelength $\lambda$ gives:

$$
\begin{aligned}
\frac{d n}{d \lambda} & =-(\lambda / n)\left[\frac{C_{2}}{\left(\lambda^{2}-C_{3}\right)^{2}}+C_{4}\right] \\
\frac{d^{2} n}{d \lambda^{2}} & =\frac{-1}{n}\left[\frac{C_{2}\left(\left(\lambda^{2}-C_{3}^{2}\right)-4 \lambda^{2}\right)}{\left(\lambda^{2}-C_{3}^{2}\right)^{3}}-C_{4}\right],
\end{aligned}
$$

The switching voltage $\mathrm{V}_{\pi}$ or the voltage required to change the output light intensity from its maximum value to its minimum value can be expressed as the following [13-15]:

$$
V_{\pi}=\frac{\lambda d}{2 \Gamma n^{3} r_{41} L_{m}},
$$

Where $\lambda$ is the operating optical signal wavelength in $\mu \mathrm{m}$, $\Gamma$ is the confinement factor, $d$ is the modulator thickness in $\mu \mathrm{m}, \mathrm{L}_{\mathrm{m}}$ is the modulator length in $\mu \mathrm{m}$, and $\mathrm{r}_{41}$ is the electrooptic coefficient for used semiconductor material.

Under the perfect velocity matching condition[16,17], achievable modulation bandwidth $\mathrm{f}_{\mathrm{m}}$ is:

$$
f_{m}=\frac{6.84}{\alpha L_{m}}, \mathrm{GHz}
$$

Where $\alpha$ is the power absorption coefficient in $\mathrm{dB} / \mu \mathrm{m}$.

Therefore the device performance index (DPI) can be expressed as the following expression $[18,19]$ :

$$
D P I=\frac{f_{m}}{V_{\pi}}, G H z / \text { Volt }
$$

If a modulating voltage $\mathrm{V}_{\mathrm{m}}$ in $\mathrm{z}$-direction is applied, the change in index for the TM polarization is:

$$
\Delta n_{e}=\frac{0.5 V_{m} n^{3} r_{41}}{L_{m}}
$$

Therefore the product of the sensitivity and the bandwidth is not related to the signal quality factor and is given by [20]:

$$
S B P=\frac{0.65 n^{2} \Gamma r_{41} c}{d \lambda}
$$

Where $r_{41}$ is the electrooptic coefficient for aluminum gallium arsenide material based EO modulator devices.

The total system rise time is the square root of the sum of the squares of the transmitter, optical fiber connection, and receiver rise times. That is given by[21]:

$$
\tau_{s}=\sqrt{\tau_{t}^{2}+\tau_{\text {mat. }}^{2}+\tau_{r}^{2}}
$$

The material dispersion time of the single mode fiber $\tau_{\text {mat. }}$. which is given by the following equation:

$$
\tau_{\text {mat. }}=-\left(\frac{L_{m} \cdot \Delta \lambda \cdot \lambda}{c}\right) \cdot\left(\frac{d^{2} n}{d \lambda^{2}}\right)
$$

In addition to providing sufficient power to the receiver, the system must also satisfy the bandwidth requirements imposed by the rate at which data are transmitted. A convenient method of accounting for the bandwidth is to com- 
bine the rise times of the various system components and compare the result with the rise time needed for the given data rate and pulse coding scheme. The system rise time is given in terms of the data rate for non return to zero pulse code by the expression[22]:

$$
B_{R}(N R Z)=\frac{0.7}{\tau_{s}},
$$

Then the bandwidth length product within electrooptic modulator device is given by $[23,24]$ :

$$
P_{R}(N R Z)=B_{R} \cdot L_{m}, \text { Gbit. } \mu \mathrm{m} / \mathrm{sec}
$$

The bandwidth for single mode operation within electrooptic modulator length $\mathrm{L}_{\mathrm{m}}$ is given by:

$$
\text { B. } W_{\text {sig. }}=\frac{0.44}{\tau_{s} \cdot L_{m}}, \mathrm{GHz}
$$

The signal to noise ratio (SNR) is a measure of signal quality at the receiver side, it is given by [25]:

$$
\begin{gathered}
S N R=\frac{\left(G P_{0} \rho\right)^{2} R_{L}}{4 k T B . W_{\text {sig. }}+2 e R_{L} B \cdot W_{\text {sig. }} G^{n}\left(I_{D}+\rho P_{0}\right)} \\
(S N R)_{d B}=10 \log S N R,
\end{gathered}
$$

Where $\mathrm{P} 0$ is the received or output optical power, $\rho$ is the detector's unamplified responsivity, $\mathrm{G}$ is the detector gain if an avalanche photodiode(APD) is used, $\mathrm{n}$ accounts for the excess noise of the APD (usually between 2,3), B.Wsig. is the signal bandwidth at the receiver, $\mathrm{k}$ is Boltzmann's con$\operatorname{stant}(\mathrm{k}=1.38 \times 10-23 \mathrm{~J} / \mathrm{K})$, e is the magnitude of the charge on an electron(1.6 $\times 10-19$ coulomb), $\mathrm{T}$ is the ambient temperature in K, ID is the detector's dark current, and RL is the resistance of the load resistor that follows the photodetector. The maximum transmission bit rate or capacity according to modified Shannon technique is given by[26,27]:

$$
B_{R(S H)}=B \cdot W_{\text {sig. }} \log _{2}(1+S N R)
$$

Where B.Wsig. is the actual bandwidth of the optical signal, and SNR is the signal to noise ratio in absolute value (i. e., not in $\mathrm{dB}$ ). Where the Shannon Bit rate length product can be given by:

$$
P_{S H}=B_{R(S H)} \cdot L_{m}
$$

\section{Simulation Results and Performance Analysis}

We have investigated ultra high speed semiconductor electrooptic modulator devices for gigahertz system operation over wide range of the affecting operating parameters as shown in Table 1.

Based on the model equations analysis, assumed set of the operating parameters, and the set of the Figs.(1-14), the following facts are assured as the following results:

\begin{tabular}{|c|c|c|}
\hline Parameter & Definition & Value and unit \\
\hline $\mathrm{T}$ & Ambient temperature & $300 \mathrm{~K} \leq \mathrm{T} \leq 330 \mathrm{~K}$ \\
\hline $\mathrm{L}_{\mathrm{m}}$ & Modulator length & $2 \mu \mathrm{m} \leq \mathrm{L}_{\mathrm{m}} \leq 5 \mu \mathrm{m}$ \\
\hline d & Modulator thickness & $\begin{array}{c}0.1 \mu \mathrm{m} \leq \mathrm{w} \leq 0.5 \\
\mu \mathrm{m}\end{array}$ \\
\hline$\tau_{\mathrm{t}}$ & Rise time of the transmitter & $0.8 \mathrm{nsec}$ \\
\hline$\tau_{\mathrm{r}}$ & Rise time of the receiver & $1 \mathrm{nsec}$ \\
\hline $\mathrm{P}_{0}$ & Output power & $0.1 \leq \mathrm{P}_{0}$, Watt $\leq 0.6$ \\
\hline $\mathrm{T}_{0}$ & Room temperature & $300 \mathrm{~K}$ \\
\hline$\lambda$ & signal operating wavelength & $\begin{array}{c}1.3 \mu \mathrm{m} \leq \lambda \leq 1.65 \\
\mu \mathrm{m}\end{array}$ \\
\hline$\Delta \lambda$ & $\begin{array}{l}\text { Spectral line width of the } \\
\text { optical source }\end{array}$ & $0.1 \mathrm{~nm}$ \\
\hline $\mathrm{r}_{41}$ & $\begin{array}{l}\text { Electoptic coefficient for } \\
\qquad \mathrm{Al}_{\mathrm{x}} \mathrm{Ga}_{(1-\mathrm{x})} \mathrm{As}\end{array}$ & $18 \mathrm{Pm} /$ volt \\
\hline $\mathrm{L}$ & Inductance & $20 \mu \mathrm{H}$ \\
\hline $\mathrm{C}$ & Capacitance & $0.2 \mathrm{pF}$ \\
\hline $\mathrm{R}_{\mathrm{L}}$ & Load resistance & $5 \mathrm{k} \Omega$ \\
\hline $\mathrm{G}$ & Detector gain & $20 \mathrm{~dB}$ \\
\hline$\alpha$ & Power absorption coefficient & $0.1-0.5 \mathrm{~dB} / \mu \mathrm{m}$ \\
\hline$\Delta \mathrm{n}_{\mathrm{e}}$ & $\begin{array}{c}\text { Effective refractive index } \\
\text { difference }\end{array}$ & $0.01 \leq \Delta \mathrm{n}_{\mathrm{e}} \leq 0.09$ \\
\hline$\Gamma$ & Confinement factor & $0.8 \leq \quad \Gamma \leq 0.95$ \\
\hline $\mathrm{I}_{\mathrm{D}}$ & Detector dark current & $8 \mathrm{nA}$ \\
\hline$\rho$ & detector responsivity & $0.8 \mathrm{~A} / \mathrm{Watt}$ \\
\hline$\eta$ & Modulator efficiency & $90 \%$ \\
\hline $\mathrm{c}$ & Speed of light & $3 \times 10^{8} \mathrm{~m} / \mathrm{sec}$ \\
\hline
\end{tabular}

i) As shown in the series of Figs. (1-3), as both modulator length and confinement factor increase, and operating optical signal wavelength decreases, this results in decreasing of switching voltage.
Table 1. Proposed operating parameters for our suggested electrooptic modulator device

ii) Fig. 4 has assured that as both modulator length and relative refractive index difference increase, this leads to increase in modulating voltage.

iii) As shown in Fig. 5 has demonstrated that as both power absorption coefficient and modulator length increase, this results in decreasing of modulation device bandwidth.

iv) Figs. $(6,7)$ have indicated that as both operating optical signal wavelength and modulator thickness increase, and confinement factor decreases, this leads to decrease in device performance index.

v) As shown in Fig. 8 has assured that as operating optical signal wavelength increases and confinement factor decreases, this results in decreasing of device sensitivity bandwidth product.

vi) Fig. 9 has proved that as both modulator length and optical output power increase, this results in increasing of signal to noise ratio.

vii) As shown in Figs. $(10,11)$ have demonstrated that as both modulator length and output power increase, this results in increasing of transmission bit rate length product. As well as modulator length increases, and output power decreases, this leads to decrease in transmission bit rates with Shannon transmission technique.

viii) Fig. 12 has indicated that as ambient temperature decreases operating optical signal wavelength increases, this leads to increase in transmitted signal bandwidth.

ix) Figs. $(13,14)$ have demonstrated that as both modulator length and operating optical signal wavelength increase, and ambient temperature decreases, this results in increasing transmission bit rate length product. As well as both modu- 
lator length and ambient temperature increase, and operating optical signal wavelength decreases, this results in decreasing of transmission bit rates with non return to zero coding formats.

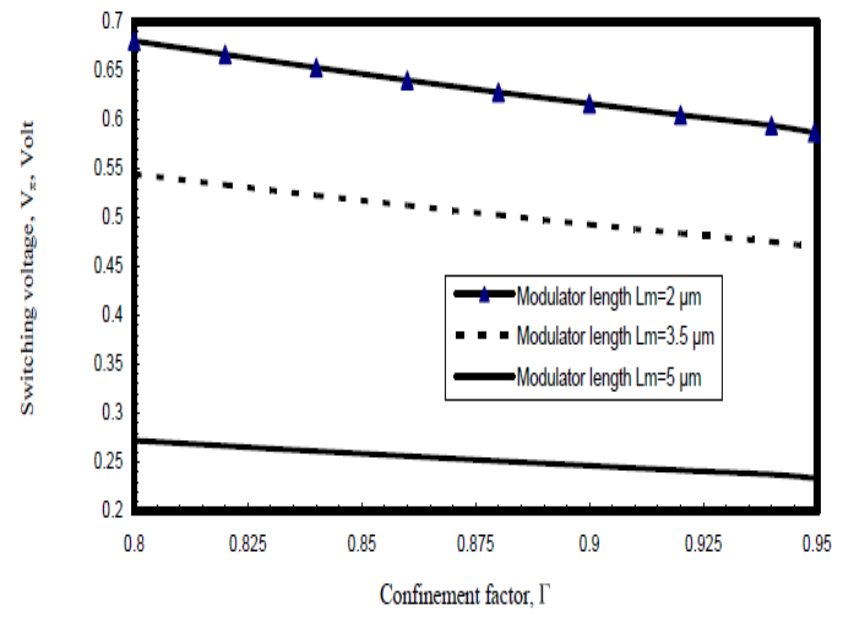

Figure 1. Variations of switching voltage versus confinement factor at the assumed set of parameters

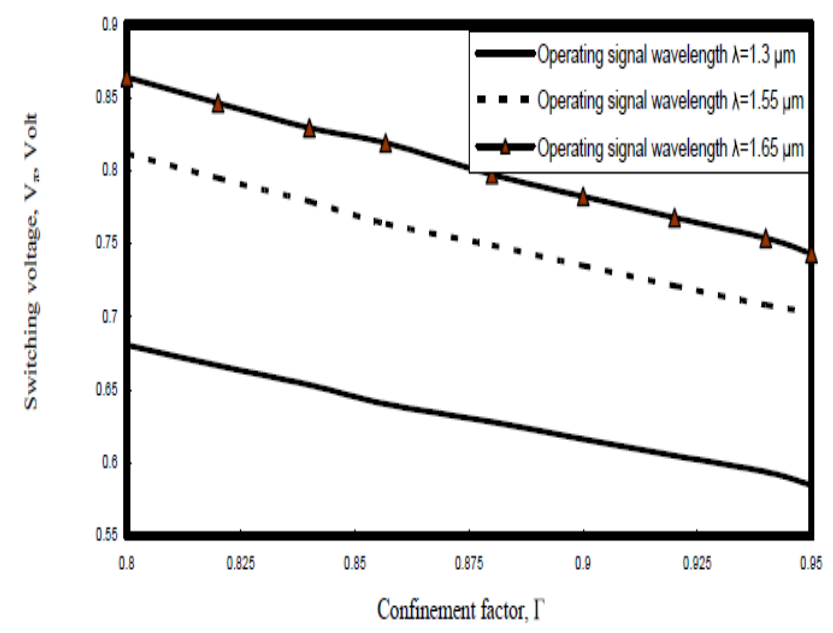

Figure 2. Variations of switching voltage versus confinement factor at the assumed set of parameters

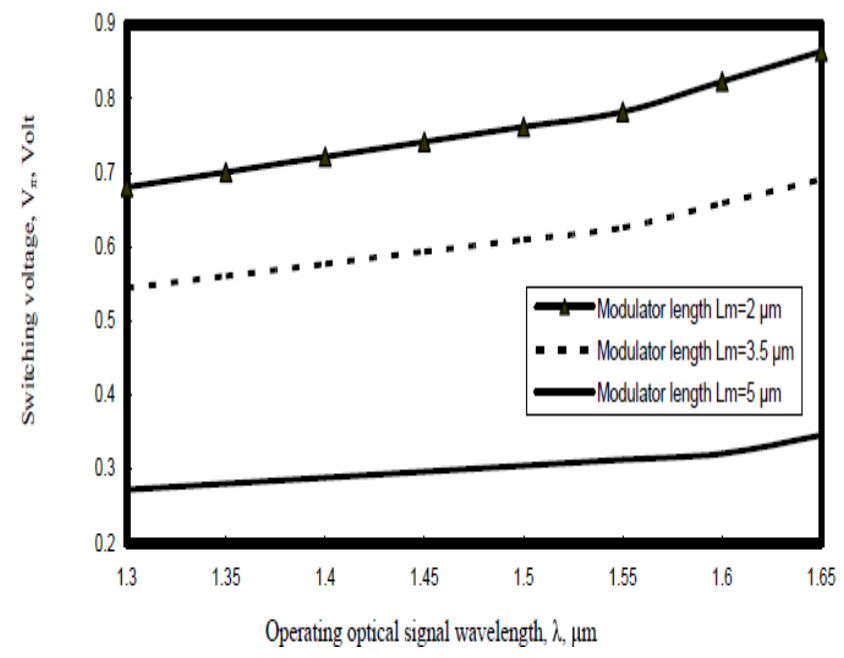

Figure 3. Variations of switching voltage versus signal wavelength at the assumed set of parameters

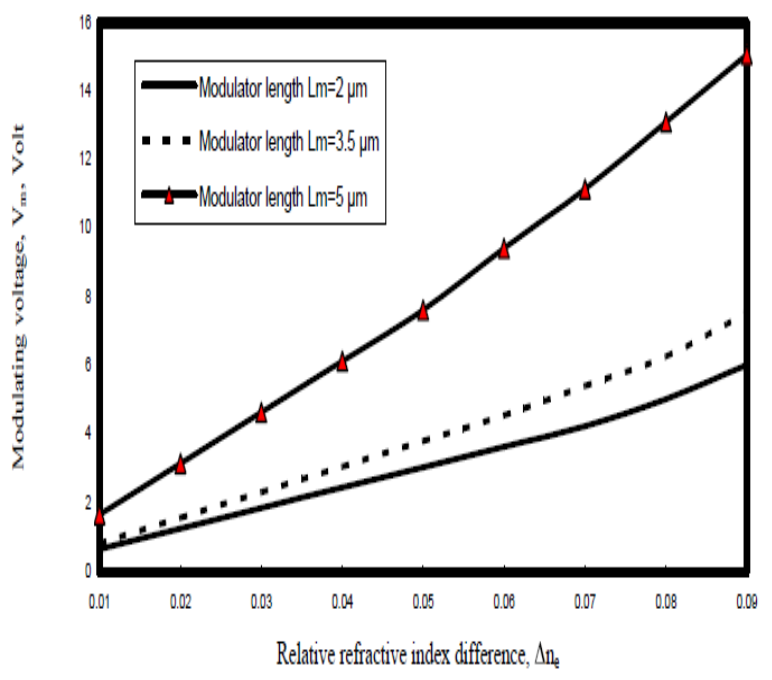

Figure 4. Variations of modulating voltage against relative index difference at the assumed set of parameters

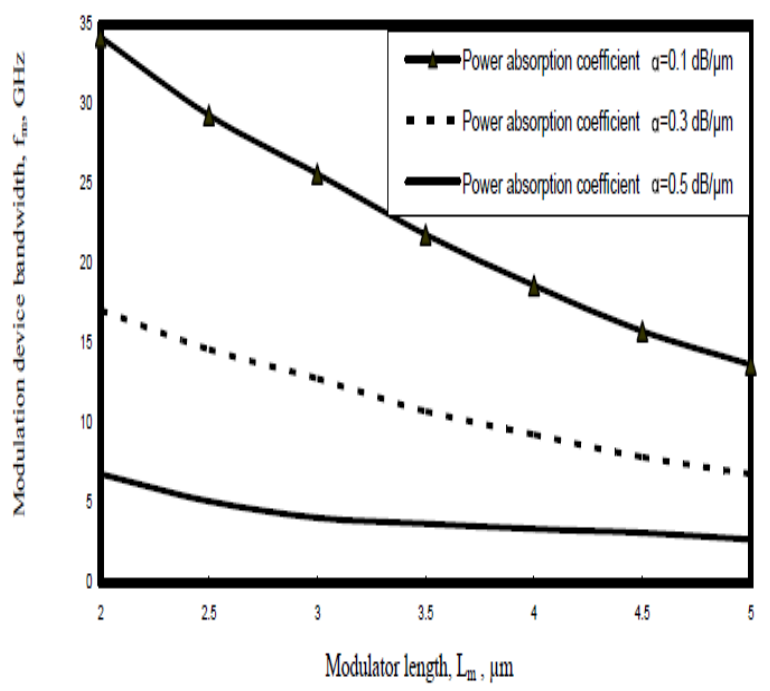

Figure 5. Variations of Modulation bandwidth against modulator length at the assumed set of parameters

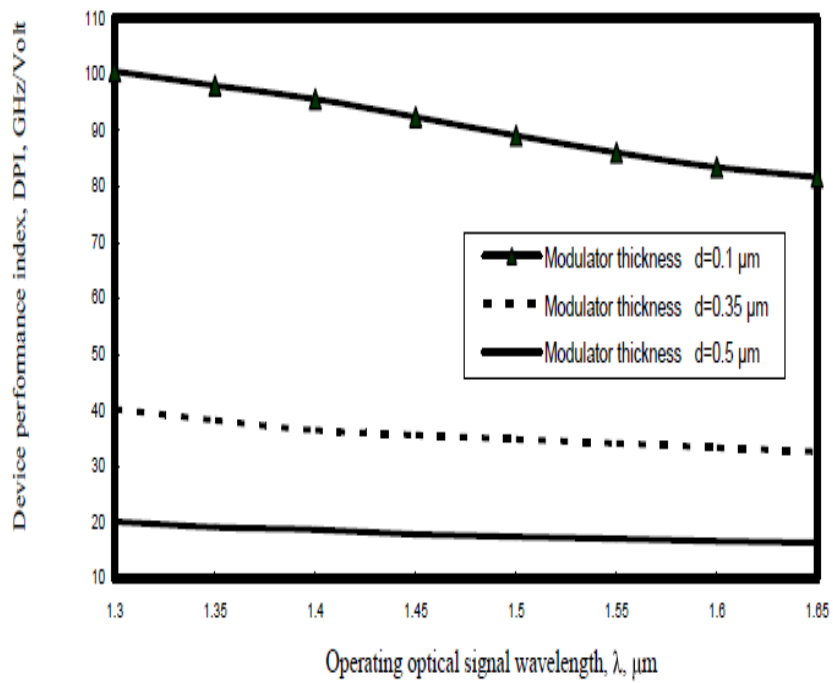

Figure 6. Variations of Device performance index against optical signal wavelength at the assumed set of parameters 


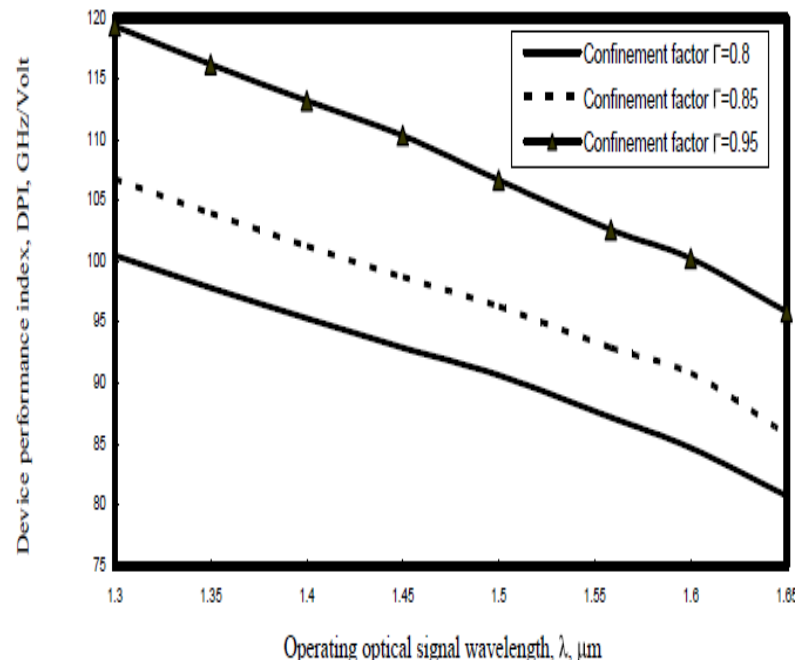

Figure 7. Variations of Device performance index against optical signal wavelength at the assumed set of parameters

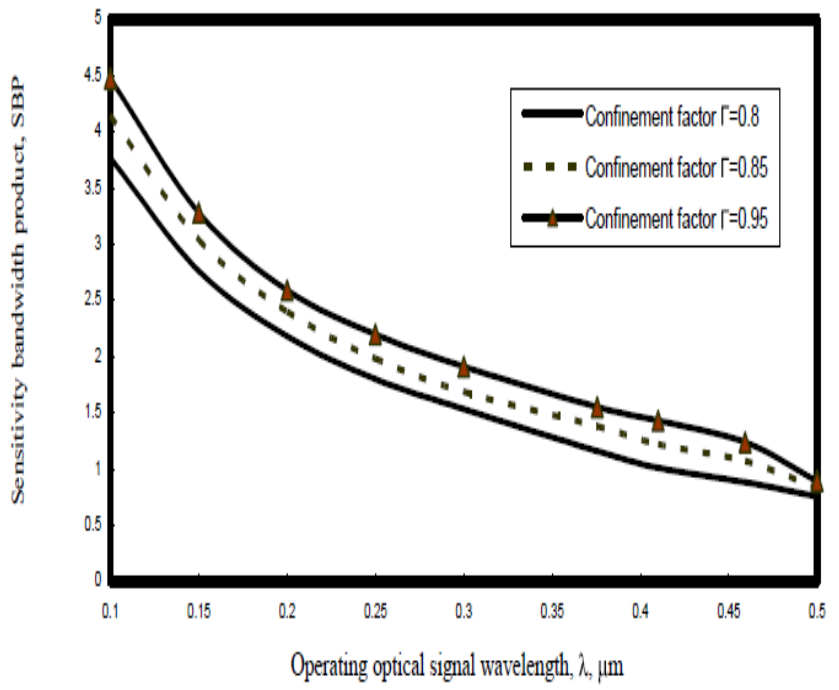

Figure 8. Variations of sensitivity bandwidth product against optical signal wavelength at the assumed set of parameters

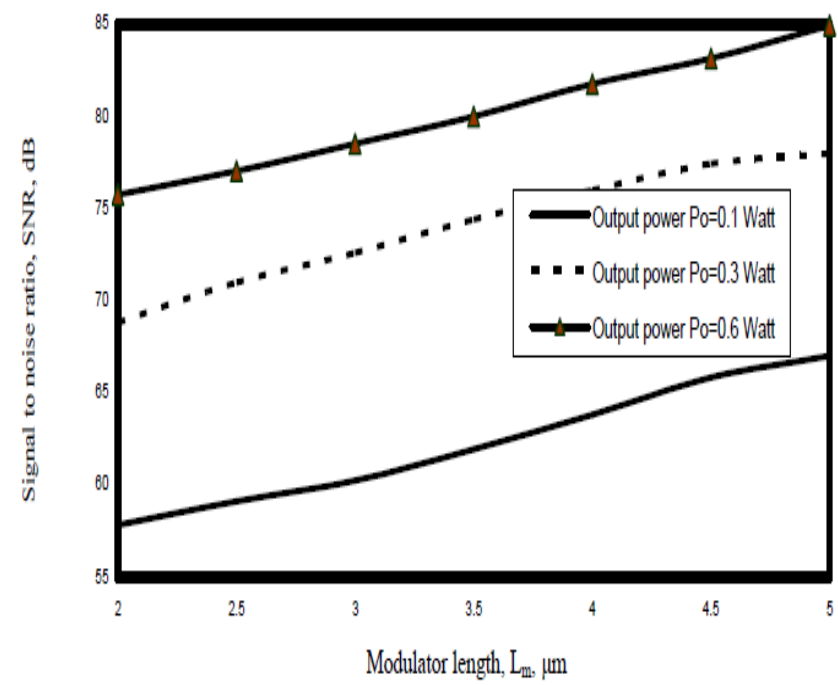

Figure 9. Variations of signal to noise ratio against modulator length at the assumed set of parameters

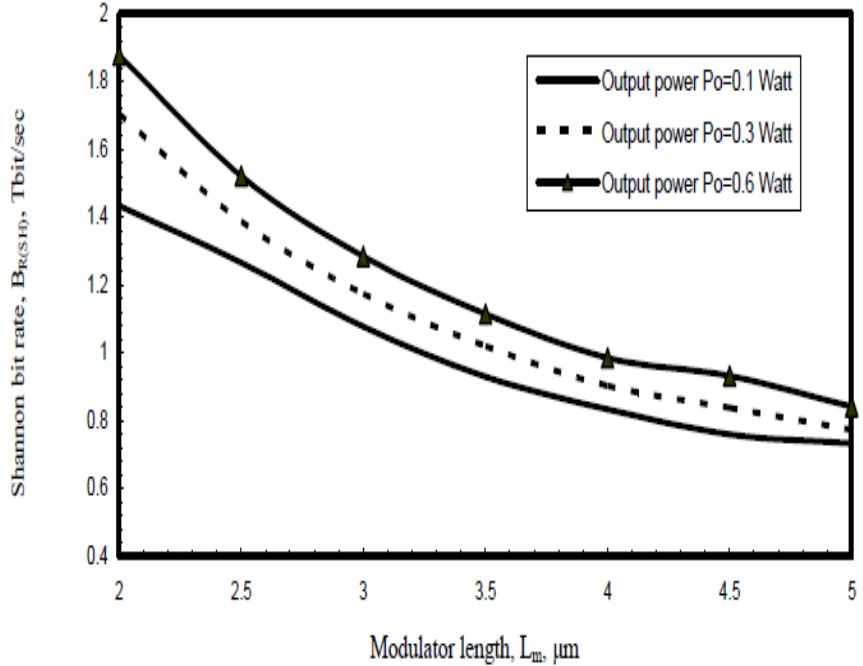

Figure 10. Variations of Shannon transmission bit rate against modulator length at the assumed set of parameters

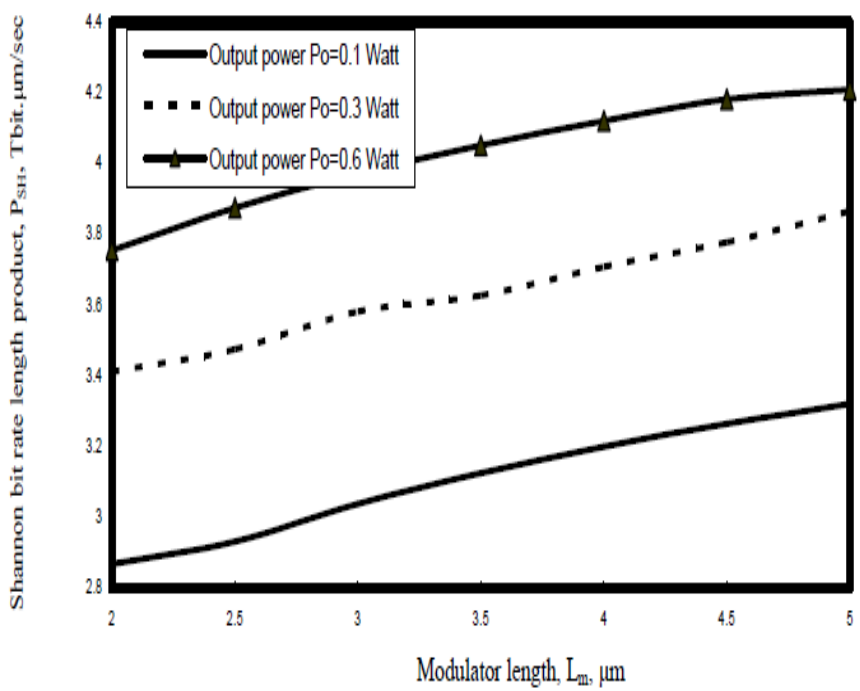

Figure 11. Variations of Shannon bit rate length product against modulator length at the assumed set of parameters

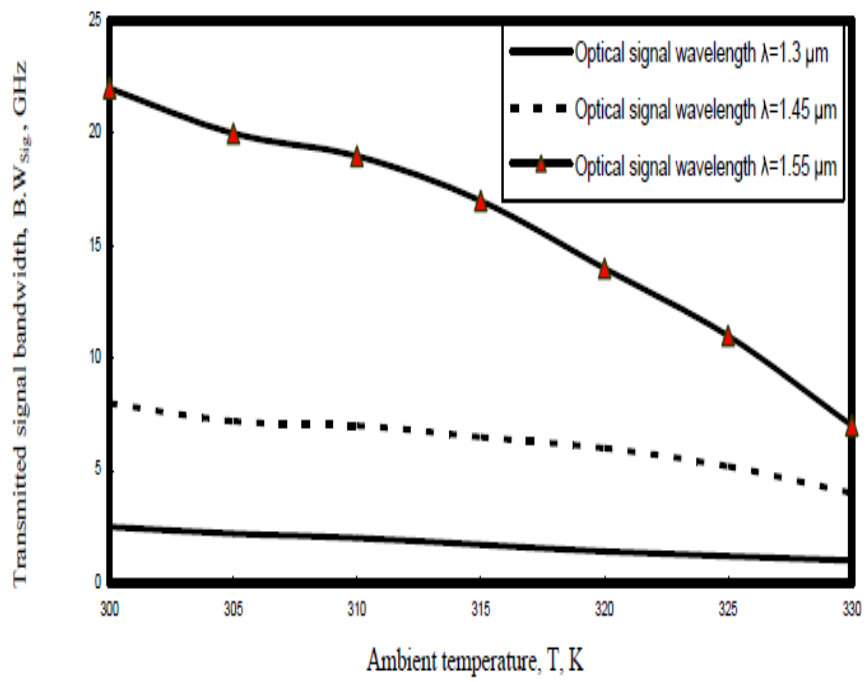

Figure 12. Variations of transmitted signal bandwidth versus ambient temperature at the assumed set of parameters 


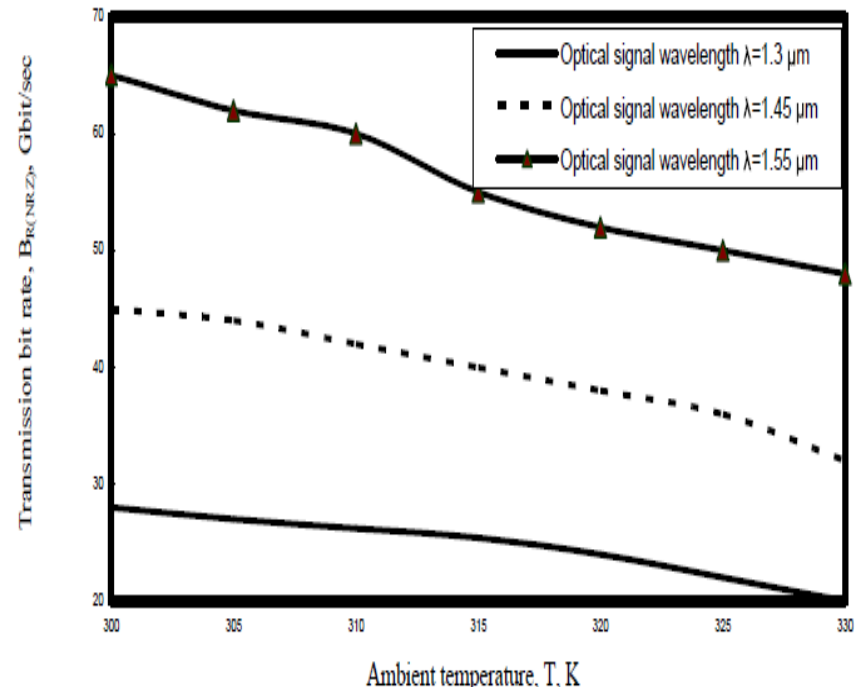

Figure 13. Variations of transmission bit rate versus ambient temperature at the assumed set of parameters

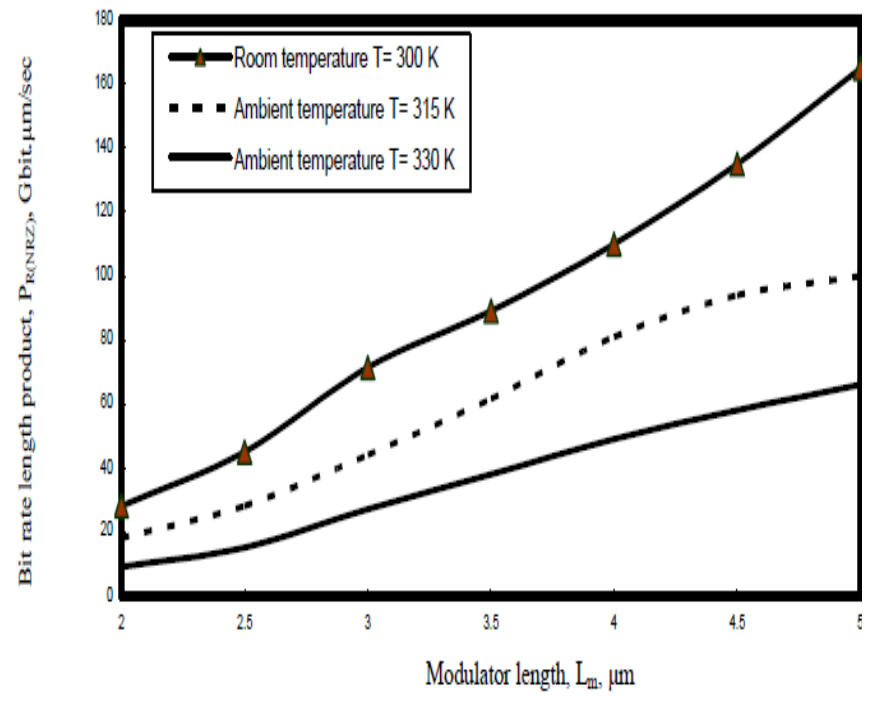

Figure 14. Variations of transmission bit rate length product against modulator length at the assumed set of parameters

\section{Conclusions}

In a summary, we have presented ultra high speed semiconductor electrooptic modulator devices for multi gigahertz operation systems. It is theoretically found that the increased confinement factor and modulator length, and the decreased operating optical signal wavelength, this leads to the decreased switching device voltage. It is evident that the decreased operating optical signal wavelength and modulator thickness, and the increased confinement factor, this results in increasing of device performance index. As well as the increased output received power and modulator length, and the increased operating optical signal wavelength, and the decreased ambient temperature, this results in the increased transmission bit rate length product with using both Shannon transmission technique and NRZ coding formats.

\section{REFERENCES}

[1] R. Soref, "Silicon Photonics Technology: Past, Present, and Future,” IEEE J. Sel. Topics Quantum Electron., Vol. 12, No. 6, pp. 1678-1687, Nov./Dec. 2006

[2] A. Liu, R. Jones, L. Liao, D. S. Rublo, D. Rubin, O. Cohen, R. Nicolaescu, and M. Paniccia, "A high Speed Silicon Optical Modulator Based on A metal Oxide Semiconductor Capacitor," Nature Photonics, Vol. 42, No. 7, pp. 615-617, 2004

[3] L. Liao, D. Samara-Rubio, M. Morse, A. Liu, D. Hodge, D. Rubin, U. D. Keil, and T. Franck, "High Speed Silicon Mach-Zehnder Modulator," Opt. Exp., Vol. 13, No.3, pp. 3129-3135, 2005

[4] L. Gu, W. Jiang, X. Chen, L. Wang, and R. T. Chen, "High Speed Silicon Photonic Crystal Waveguide Modulator for Low Voltage Operation,” Appl. Phys. Lett., Vol. 90, No. 2, pp. $5-8,2007$

[5] H. Kim and A.H. Gnauck, "Chirp Characteristics of Dual Drive Mach-Zehnder Modulator with A finite DC Extinction Ratio," IEEE Photonics Technol. Lett., Vol.14, No.3, pp. 298-300, 2002

[6] K. Tsuzuki, H. Yasaka, T. Ishibashi, T. Ito, S. Oku, R. Iga, Y. Kondo, and Y. Tohmori, "10-Gbit/s, 100-km SMF Transmission Using an InP Based n-i-n Mach-Zehnder Modulator with A driving Voltage of 1.0 Vpp," Proc. Optical Fiber Communication 2004 (OFC'04) Postdeadline Papers, 2004

[7] K. Tsuzuki, T. Ishibashi, T. Ito, S. Oku, Y. Shibata, R. Iga, Y. Kondo, and Y. Tohmori, "40 Gbit/s n-i-n InP Mach-Zehnder Modulator with a peak voltage of $2.2 \mathrm{~V}$," Electron. Lett., Vol.39, No.20, pp.1464-1466, 2003

[8] K. Tsuzuki, T. Ishibashi, T. Ito, S. Oku, Y. Shibata, T. Ito, R. Iga, Y. Kondo, and Y. Tohmori, "1.6 V-driven 40-Gbit/s n-i-n Mach-Zehnder modulator based on InP substrate," Proc. 9th Optoelectronics and Communication Conference 2004 (OECC'04), 15E3-2, pp.706-707, 2004

[9] K. Tsuzuki, T. Ishibashi, T. Ito, S. Oku, Y. Shibata, T. Ito, R. Iga, Y. Kondo, and Y. Tohmori, "A 40-Gb/s InGaA1As-InAlAs MQW n-i-n Mach-Zehnder Modulator with A drive Voltage of 2.3 V," IEEE Photon. Technol. Lett., Vol. 17, No. 1, pp. 46-48, Jan. 2005

[10] Y. Cui and P. Berini, "Modeling and Design of GaAs Traveling Wave Electrooptic Modulators Based on the Planar Microstrip Structure," J. Lightw. Technol., Vol. 24, No. 6, pp. 2368-2378, Jun. 2006

[11] J. Shin, S. Wu, and N. Dagli, "35-GHz Bandwidth, 5-V-cm Drive Voltage, Bulk GaAs Substrate Removed Electrooptic modulators," IEEE Photon. Technol. Lett., Vol. 19, No. 18, pp. 1362-1364, Sep. 2007

[12] Ibrahim M. El-dokany, Abd El-Naser A. Mohammed, Ahmed Nabih Zaki Rashed and Mahomud M. Eid, "Ultra Wide Wavelength Multiplexing/Demultiplexing Conventional Arrayed Waveguide Grating (AWG) Devices for Multi Band Applications," International Journal of Computational Intelligence and Information Security, Vol. 2, No. 2, pp. 20-32, Feb. 2011 
[13] Abd El-Naser A. Mohammed, Ahmed Nabih Zaki Rashed and Mahomud M. Eid, "Rapid Progress of a Thermal Arrayed Waveguide Grating Module for Dense Wavelength Division Multiplexing Applications," International Journal of Computational Intelligence and Information Security, Vol. 2, No. 2, pp. 39-50, Feb. 2011

[14] B.-J. Seo and H. R. Fetterman, "True-time-delay element in lossy environment using EO waveguides," IEEE Photon. Technol. Lett., Vol. 18, No. 1, pp. 10-12, Jan. 2006

[15] Abd El-Naser A. Mohammed, Abd El-Fattah A. Saad, and Ahmed Nabih Zaki Rashed, "Matrices of the Thermal and Spectral Variations for the fabrication Materials Based Arrayed Waveguide Grating Devices," International Journal of Physical Sciences, Vol. 4, No. 4, pp. 205-211, April 2009

[16] Allocation and Service Rules for the $71-76 \mathrm{GHz}, 81-86 \mathrm{GHz}$, and $92-95 \mathrm{GHz}$ Bands, Federal Communications Commission, Washington, DC, Mar. 2005

[17] Abd El-Naser A. Mohammed, Abd El-Fattah A. Saad, and Ahmed Nabih Zaki Rashed,"Thermal Sensitivity Coefficients of the Fabrication Materials Based A thermal Arrayed Waveguide Grating (AWG) in Wide Area Dense Wavelength Division Multiplexing Optical Networks," International Journal of Engineering and Technology (IJET), Vol. 1, No. 2, pp. 131-139, June 2009

[18] Abd El-Naser A. Mohammed, Abd El-Fattah A. Saad, and Ahmed Nabih Zaki Rashed, "Characteristics of the Fabrication Materials Based Arrayed Waveguide Grating (AWG) in Passive Optical Networks (PONs), " International Journal of Material Sciences Research, Vol. 1, No. 6, pp. 89-97, June 2009

[19] H. Tazawa and W. H. Steier, "Analysis of Ring Resonator Based Traveling Wave Modulators," IEEE Photon. Technol. Lett., Vol. 18, No. 1, pp. 211-213, Jan. 2006

[20] Abd El-Naser A. Mohammed, Abd El-Fattah A. Saad, and Ahmed Nabih Zaki Rashed*, "Study of the Thermal and Spectral Sensitivities of Organic-Inorganic Fabrication Materials Based Arrayed Waveguide Grating for Passive Optical Network Applications," Journal of Engineering and Technology Research, Vol. 1, No. 5, pp. 81-90, Aug. 2009
[21] G. Xu, Z. Liu, J. Ma, B. Liu, S.-T. Ho, L. Wang, P. Zhu, T. J. Marks, J. Luo, and A. K. Jen, "Organic Electro-optic Modulator Using Transparent Conducting Oxides as Electrodes," Opt. Expr., Vol. 13, No. 2, pp. 7380-7385, Sep. 2005

[22] Abd El-Naser A. Mohammed and Ahmed Nabih Zaki Rashed, "Ultra Wide Band (UWB) of Optical Fiber Raman Amplifiers in Advanced Optical Communication Networks," Journal of Media and Communication Studies, Vol. 1, No. 4, pp. 56-78, Oct. 2009

[23] Abd El-Naser A. Mohammed, Mohammed A. Metawe'e, Ahmed Nabih Zaki Rashed, and Mohamoud M. Eid "Distributed Optical Raman Amplifiers in Ultra High Speed Long Haul Transmission Optical Fiber Telecommunication Networks," IJCNS International Journal of Computer and Network Security, Vol. 1, No. 1, pp. 1-8, Oct. 2009

[24] Abd El-Naser A. Mohammed, Mohammed A. Metawe'e, Ahmed Nabih Zaki Rashed, and Amina M. El-nabawy "Unguided Nonlinear Optical Laser Pulses Propagate in Waters With Soliton Transmission Technique," IJMSE International Journal of Multidisciplinary Sciences and Engineering, Vol. 2, No. 1, pp. 1-10, March 2011

[25] M. Michalak, Y. Kuo, F. Nash, A. Szep, J. Caffey, P. Payson, F. Haas, B. Mckeon, P. Cook, G. Brost, J. Luo, A. Jen, L. Dalton, and W. Steier, "High Speed Polymer Modulator," IEEE Photon. Technol. Lett., Vol. 18, No. 11, pp. 1207-1209, Jun. 2006

[26] Abd El-Naser A. Mohammed, Mohamed M. E. El-Halawany, Ahmed Nabih Zaki Rashed, and Mohammed S. F. Tabour "High Transmission Performance of Radio over Fiber Systems over Traditional Optical Fiber Communication Systems Using Different Coding Formats for Long Haul" International Journal of Computer Science and Telecommunications (IJCST), Vol. 2, No. 3, pp. 29-42, June 2011

[27] Abd El-Naser A. Mohammed, Abd El-Fattah A. Saad, Ahmed Nabih Zaki Rashed, and Hazem M. Hageen "Low Performance Characteristics of Optical Laser Diode Sources Based on NRZ Coding Formats under Thermal Irradiated Environments" International Journal of Computer Science and Telecommunications (IJCST), Vol. 2, No. 2, pp. 20-30, April 2011 